\title{
Frontières
}

\section{La souffrance des soignants en soins palliatifs}

\section{Dominique Jacquemin}

Volume 17, numéro 2, printemps 2005

Surtout, ne pas souffrir

URI : https://id.erudit.org/iderudit/1073486ar

DOI : https://doi.org/10.7202/1073486ar

Aller au sommaire du numéro

Éditeur(s)

Université du Québec à Montréal

ISSN

1180-3479 (imprimé)

1916-0976 (numérique)

Découvrir la revue

Citer cet article

Jacquemin, D. (2005). La souffrance des soignants en soins palliatifs. Frontières, 17(2), 28-33. https://doi.org/10.7202/1073486ar

\section{Résumé de l'article}

Les soins palliatifs doivent répondre, en France, à une obligation de moyens dans la prise en charge des personnes en fin de vie. Faisant ainsi partie de l'imaginaire social, ils pourraient sembler en adéquation avec la volonté de nombreux soignants d'assumer au mieux cette ultime période de la vie. Cependant, un idéal - qui n'est pas à remettre en question - se trouve confronté aux limites du " bien faire » dans les pratiques. Divers décalages ont à être pensés et nommés pour reconnaître, rencontrer et accompagner la souffrance des soignants engagés dans ce type de soins. 


\section{Résumé}

Les soins palliatifs doivent répondre, en France, à une obligation de moyens dans la prise en charge des personnes en fin de vie. Faisant ainsi partie de l'imaginaire social, ils pourraient sembler en adéquation avec la volonté de nombreux soignants d'assumer au mieux cette ultime période de la vie. Cependant, un idéal - qui n'est pas à remettre en question se trouve confronté aux limites du «bien faire» dans les pratiques. Divers décalages ont à être pensés et nommés pour reconnaître, rencontrer et accompagner la souffrance des soignants engagés dans ce type de soins.

Mots clés: soins palliatifs - souffrance morale - éthique - limite.

\begin{abstract}
In France, palliative care should meet a "means obligation" in taking charge of people at the end of their life. Being part of social imaginary, it could seem adequate to the willingness of the majority of the carers to address as best as possible this ultimate period of life. However, this aspiration - that we absolutely not questioning here - could are meet many obstacles in practices. This distance between theory and practice need to be named and reflected in order to recognize in their suffering, address and sustain the carers engaged in this kind of care.
\end{abstract}

Keywords: palliative care - suffering moral - ethics - limits.

\section{La souffrance des soignants en soins palliatifs}

\author{
Dominique Jacquemin, Ph.D., \\ Centre d'éthique médicale, \\ Université catholique de Lille.
}

Envisager l'existence d'une souffrance des soignants exerçant en soins palliatifs peut sembler paradoxal tant ces derniers seraient volontiers considérés, au cœur d'un imaginaire social et médical, comme les spécialistes de la rencontre de la souffrance de l'autre, formés à cet effet et généralement situés comme professionnels dans des lieux «privilégiés», équipes mobiles ou unités de soins palliatifs dont certains ne manquent pas de souligner le «luxe» en termes de normes de personnel et de modalités d'accompagnement, particulièrement au regard de la souffrance psychique (Marin, 2004, p. 60-63). Et pourtant, comme tout humain, les soignants en soins palliatifs, confrontés à la rencontre de l'autre gravement malade, n'échappent pas à la souffrance, réalité que nous identifions ici en ces termes: une expérience subjective se situant entre malaise et mal-être personnel et professionnel, fondée sur la conviction d'un inaccomplissement entre une visée du bien et sa possible réalisation.

C'est bien pour donner droit à cette souffrance, trop facilement considérée comme mal venue à mettre au jour, que nous nous risquons à ces quelques lignes afin de restaurer ces soignants dans cette capacité à souffrir et à nommer cette expérience. Trop souvent forclue au nom d'un idéal du soin, cette souffrance a pourtant à se dire pour pouvoir accompagner celle du plus souffrant encore qu'est le malade, tant nous croyons que cette dernière se doit d'être exorcisée pour être vécue et assumée en humanité: «[...] il s'agit en vérité d'apprivoiser l'innommable pour ne plus être «agi» par lui. Tant que l'on ne s'est pas réapproprié le mal, tant que l'on n'est pas parvenu à le nommer pour l'inscrire dans sa vie, tant que l'on n'a pas trouvé la force de le regarder en face (nommer, c'est mettre à distance) et de dire "ça m'est arrivé", il continue à distiller son poison, à maintenir ouverte la blessure» (Longneaux, 2004, p. 8). Cette conviction, nous l'avons découverte dans notre propre expérience d'éthique clinique telle qu'elle se trouve mise en œuvre au Centre d'éthique médicale de Lille, consistant à accompagner des équipes soignantes dans un processus de relecture a posteriori de situations de soins difficiles, particulièrement dans leur dimension éthique (Cadoré, 1997, p. 24-33).

Il nous semble important de replacer la question dans le contexte de la rencontre soignante. Et ce soin ne se joue pas dans un cadre d'exercice neutre: les soins palliatifs ont dû s'imposer au cœur d'une médecine objective et technique, ce qui n'est pas sans conséquence quant à la visée du bien qu'ils poursuivent et qui leur est aujourd'hui socialement demandé. Ce premier constat nous conduira à envisager plus largement certaines contraintes liées au fonctionnement hospitalier et qui modèlent tant la rencontre avec l'autre souffrant que l'appréciation qui peut en être faite. Ces deux premiers temps de l'analyse devraient nous permettre de mettre au jour un triple niveau de souffrance possible pour les soignants en soins palliatifs: une souffrance psychique et morale liée à un excès de responsabilité professionnelle prenant appui sur une visée du «bien faire » propre aux soins palliatifs. Au terme de ce parcours, nous aurons à nous demander comment il est possible de restaurer pleinement le soignant dans sa posture d'humanité. 


\section{LA SOUFFRANCE}

\section{AU CCEUR D'UNE RENCONTRE}

On ne peut ouvrir un questionnement relatif à une souffrance spécifique des soignants en soins palliatifs sans s'arrêter quelque peu à leur horizon d'action: la rencontre de l'autre souffrant proche de sa mort, idéal du soin survalorisé en ce lieu par l'exigence légitime d'accompagner et de soigner au mieux. Or, ces différents niveaux constitutifs de ce que nous pourrions nommer le «contrat palliatif» risquent d'être générateurs de souffrance pour le soignant.

\section{RENCONTRER UNE SOUFFRANCE}

\section{MULTIFORME}

La rencontre de l'autre souffrant n'est certes pas l'apanage des soignants de soins palliatifs, car tout soignant se trouve confronté à cette expérience. Ce qui leur est peut-être davantage propre, en soins palliatifs, ce serait le caractère paroxystique de cette souffrance, tant elle se trouve toujours liée à une expérience plus ou moins proche de la mort, ce qui lui donnerait un contenu existentiel spécifique, et le fait qu'elle se présente comme une souffrance à soutenir dans une dimension répétitive et omniprésente.

En effet, la présence d'un patient souffrant, proche de sa mort, conduit le soignant à une expérience particulière de la rencontre s'il veut effectivement prendre soin, avoir un souci de lui. Rappelons-nous dès lors quelques facettes de cette souffrance vécue par le malade. Le patient de soins palliatifs, par l'expérience de sa condition souffrante où la mort peut toujours se profiler comme dernier aboutissement, vit un processus large d'altération de soi qui se devra d'être rencontré et accompagné: "Personne alors [...] ne peut ou ne veut nous aider. Notre cri est vain et "ça ne sert à rien de parler". La parole n'est que du vent: ce qui est seulement vrai, c'est que nos repères soi-disant objectifs, repères sentimentaux, sociaux, charnels, ont disparu et nous avec » (Vasse, 1983, p. 27). Si l'impératif éthique premier sera la nécessaire compétence en matière de traitement de la douleur (Matray, 2004, p. 138-139) pour éviter tout repli douloureux du patient généralement nommé «douleur totale», le soignant restera confronté à cette femme, cet homme en effort de compréhension de lui-même; les mots risqueront de manquer pour permettre à deux humanités questionnées, celle du soigné certes mais aussi celle du soignant qui aimerait comprendre, de pouvoir se rencontrer. Il n'est effectivement pas simple de soigner lorsque, comme le souligne Denis Vasse, le soin et la souffrance deviennent lieux de médiation: «Subrepticement, dans une sorte de crispation, la souffrance devient comme la raison d'être du souffrant et du soignant, au lieu d'être l'objet médiateur de la rencontre» (Vasse, 1983, p. 45). Face à cette souffrance, toujours en tension entre sa dimension objective, support du soin et la parole dont elle pourrait être porteuse en termes de signe (Vergely, 1997, p. 58-59), il reste question de se situer comme soignant, atteint certes par la rencontre de l'autre en souffrance, mais ayant à agir: «La plus grande difficulté que rencontre probablement le soignant face à la souffrance est de ne plus savoir quoi dire, ni quoi répondre, ayant pourtant l'impression qu'il serait un déserteur s'il se taisait» (Cadoré, 1994, p. 47).

Et c'est bien ici que nous sommes confrontés à cette lourdeur spécifique de la souffrance, surtout lorsqu'elle se répète dans la prise en charge des patients car, dans cette confrontation à la souffrance, qu'est-ce qui de moi ou de l'autre est en train de se dévoiler? En effet, prendre soin de l'autre souffrant ne peut se vivre sans que l'on soit confronté à la question du mystère, celui de la rencontre certes, mais plus fondamentalement celui de l'existence, exigeant un patient rapport à une temporalité souvent décalée, trop rarement identifiée. En effet, tandis que le malade s'éprouve dans son devenir encore incertain, porteur de sa propre histoire, ses proches et les soignants s'inscrivent déjà dans un autre temps, celui du présent d'une action toujours déjà orientée vers un avenir plus ou moins proche, celui de la mort de l'autre. Il en résultera parfois un vécu douloureux, certes pour le patient incompris, mais également pour ses proches et une équipe soignante affrontés à la passivité du présent de l'autre dont il importe de prendre soin: «Accompagner une personne qui souffre ne signifie [...] pas seulement conforter dans la patience résignée face à l'épreuve; c'est aussi veiller aux côtés de quelqu'un qui fait l'expérience d'une aspiration tenace à l'unité de son identité» (Cadoré, 1994, p. 51).

\section{DANS LE SOUCI \\ D'UNE APPROCHE GLOBALE \\ DE LA PERSONNE}

C'est pour répondre au mieux à la complexité de ce que peut vivre le malade que la pratique des soins palliatifs a remis à l'honneur l'approche globale de la personne, reprenant à son compte la théorie de Virginia Henderson où il est question de pouvoir approcher le malade dans la globalité de son existence, cette dernière étant porteuse d'une diversité de besoins qu'il importe de satisfaire. S'il est heureux de se rendre compte que la personne souffrante n'est pas qu'un corps à traiter mais bien une personne inscrite socialement, ayant une psychologie, une spiritualité porteuse de valeurs y compris religieuses
(Jacquemin, 2002, p. 125 et suiv.), ce souci de rencontrer l'autre dans la globalité de son existence s'est parfois traduit, avec une visée de bien faire, par une prise en charge globale, sinon totale, comme si le soignant devait être en situation de tout porter, de tout soigner, traiter, solutionner; ce qui est manifestement impossible à réaliser.

L'exigence de répondre aux «besoins spirituels » du patient peut être illustrative de ce souci de le rejoindre dans ce qu'il vit, dans les altérations et déplacements que la maladie et l'expérience de la souffrance lui imposent, exigence positive mais source de souffrance pour le soignant si elle ne se trouve pas réfléchie et mise en perspective de ce qui est légitimement possible. Historiquement, la notion de besoin spirituel fut proposée par Virginia Henderson en 1968 dans Principes fondamentaux des soins infirmiers (Henderson, 1969, p. 14-15) où il s'agissait de reconnaître la dimension religieuse et éthique de l'homme parmi d'autres besoins fondamentaux puisqu'elle définissait ces besoins comme "pratiquer sa religion ou agir selon sa conception du bien et du mal». Et c'est dans les années 1980 que Jean Vimort, dans son ouvrage Ensemble face à la mort (Vimort, 1987), s'efforce de préciser le contenu de ces besoins spirituels, ce qu'ils peuvent recouvrir: besoin de se réconcilier avec l'existence, de reprendre des options de fond, de se libérer de la culpabilité, de retrouver des solidarités, de croire à une continuité de la vie, de se séparer dignement des siens, de croire à un au-delà de la mort Si ces sept items permettent de déblayer le chantier en évoquant des dimensions importantes, d'ordre spirituel, que le patient peut vivre, il faut en même temps reconnaître leur caractère un peu théorique, systématique et s'enracinant très peu sur le fond d'une relation de soin. Cette dimension de besoin spirituel fut beaucoup travaillée dans le cadre des soins palliatifs en tant que dimension essentielle à prendre en compte à côté des besoins biologiques, sociaux et psychologiques, besoin qui «désignerait, au cœur de la personne, le plus intime de l'intime, la personne elle-même dans son statut de sujet, celui qui la fait libre et irréductible à tout autre» (Matray, 1995, p. 18). Ici encore, si cette définition donne bien à penser qu'il existe une dimension mystérieuse, vaste, non objectivable en tout être humain, elle pourrait faire croire que tout besoin comme manque puisse être comblé et serait difficilement opératoire dans le cadre de la relation de soin. Pourtant, cette exigence de niveau de rencontre de l'autre souffrant s'est imposée dans certaines équipes au point que le soin et l'accompagnement idéals seraient ceux qui permettent de rencontrer le patient à ce niveau 
d'existence, de promouvoir les nécessaires réconciliations afin que le patient chemine au mieux vers sa fin. La question n'est pas ici de dénier l'importance de cette attention à ce que le malade peut vivre ni l'aide réelle qui peut effectivement lui être apportée mais bien d'affirmer son caractère délétère pour le soignant lorsque cette visée possible de la rencontre se traduit en impératif absolu, sans parler ici de la personne soignée pour qui cette attention à ce qu'elle vit pourrait se traduire en véritable intrusion.

\section{DANS UNE VISÉE}

\section{IDÉALISÉE DU SOIN}

Ce qui vient d'être décrit de «l'approche globale » pourrait n'être qu'une illustration d'un idéal des soins palliatifs qui, s'il n'est pas appréhendé dans une vision critique, risque sans cesse de mettre les soignants en situation d'échec et donc de souffrance au regard de ce qu'ils s'efforcent de vivre dans le soin à l'autre souffrant. Redisons-le encore, il n'est pas ici question de critiquer l'ensemble de ces soignants confrontés à un lourd exercice professionnel mais bien de pointer les difficultés, les souffrances que peut engendrer un idéal professionnel lorsqu'il n'est pas resitué de manière critique.

Si nous avons déjà largement envisagé cette problématique par ailleurs (Jacquemin, 2001), il nous semble cependant important d'en reprendre ici certains éléments au regard de la question qui nous occupe. En effet, tant les discours relatifs aux soins palliatifs qu'un idéal vécu dans le soin sont traversés par certaines visées du «bien faire»: la nécessité d'accompagner tant le patient que la famille dans un cheminement personnel qui est à faire dans la perception d'une mort prochaine, la suppression des prises en charge techniques, l'idéalisation de l'équipe comme ensemble de professionnels fondant les processus de décision, l'élucidation de la mort comme confrontation individuelle et collective - ce sont toutes ces conceptions de la «bonne mort» vers laquelle il faut tendre, l'évidence que le patient ne peut, dans son cheminement, dénier sa mort -, l'importance d'ouvrir des chemins d'avenir, de renouveau, de réconciliation pour le patient et ses proches. Ces dimensions traduisent un souci de prise en charge totalisante: rencontrer au mieux, et parfois dans une visée quelque peu volontariste, tous les éléments de vie d'une personne au terme de l'existence. Or, lorsque ces points d'attention d'une bonne pratique se muent ou sont interprétés en termes d'impératifs, les soignants ne peuvent que connaître une profonde souffrance de «ne pas y arriver», souffrance que le malade se trouvera lui aussi à vivre.

\section{LA SOUFFRANCE AU CGUR} D'UN LIEU QUI MET EN ÉCHEC

Avant d'envisager les modalités de souffrance que peuvent éprouver les soignants en soins palliatifs, il semble important de faire un détour par le lieu où s'exerce leur pratique: l'hôpital contemporain soutenant et traduisant une certaine médecine. Nous ne référons pas ici à une critique du fonctionnement de la médecine (Jacquemin, 2004a, p. 16-18), nous contentant de mettre en évidence divers traits donnant à comprendre certains décalages pouvant créer des tensions, de la souffrance chez les soignants.

\section{UNE MÉDECINE POUR GUÉRIR}

Il est aujourd'hui manifeste que la fonction et la requête médicales occupent une place centrale non seulement dans l'expérience singulière des individus mais également dans la gestion de ce qu'on pourrait appeler «le malheur social»; nous lui demandons de prendre en charge tout ce que nous vivons comme pénible à vivre, et la médecine répond. Elle répond même à nos désirs d'immortalité. Au cœur d'une société où la réalité de la mort, de la souffrance et parfois même de la maladie se trouve gommée - notre image de l'homme n'est pas celle d'un être qui peut connaître des limites -, la médecine est approchée par nos contemporains comme un lieu de salut qui pourra repousser toutes les limites. La structure hospitalière devient, dans ce contexte, l'espace par excellence où vont se croiser l'offre médicale et les sollicitations tant individuelles que sociales. Et c'est bien ce croisement qui risquera d'exclure de ce lieu l'individu souffrant, proche de sa mort, s'il est question de guérir et de guérir à tout prix. Tel est l'horizon de sens dans lequel sont formés la majorité des soignants et telle est bien d'ailleurs leur responsabilité -: guérir! Cependant, une vision de la médecine uniquement centrée sur le seul processus de guérison absolue, délié de l'existence totale du sujet soigné, risquera parfois de faire de l'acte médical, souvent ramené à sa seule dimension technique, l'unique raison d'être de l'acte de soins qui déborde largement l'unique visée de guérison. C'est ce qu'ont pu rappeler voici quelques années les premiers développements des soins palliatifs, avec la célèbre formule de Cicely Saunders: "lorsqu'il n'est plus possible de traiter (to cure), il est encore possible de soigner (to care) ». Mais cette manière de fonctionner de la médecine risque aussi d'inspirer aux individus souffrants une requête absolue à l'égard de cette même médecine: puisqu'elle se présente comme capable de guérir, c'est bien ce que j'ai à exiger d'elle, et là aussi quel qu'en soit le prix. "Dans un cas comme tout juste le temps de naître

dégringole

et s'écrase dans la mort

dans l'autre, une médecine techno-scientifique, efficace deviendra pour elle-même sa seule raison d'être. Cependant, dans l'existence singulière tant des soignants que des patients et de leur famille, force est de constater que ces objectifs "implicitement vécus" ne se trouvent pas toujours rencontrés. » (Jacquemin, 2002, p. 80.)

\section{QUAND SURVIENT L'IMPUISSANCE}

Nous le comprendrons facilement, en ces hauts lieux de la technicité, les choses deviennent tout autre lorsque la souffrance et la mort possible résistent. Le risque sera grand d'en exclure ce patient résistant - ce qui fut l'un des motifs de la genèse des soins palliatifs -, et ce d'autant plus que les soignants, essentiellement formés à l'efficacité, se trouveront en échec dans leur rapport à l'action: plus que la maladie non vaincue par une thérapeutique dans la personne du malade, c'est un peu le soignant luimême qui se trouve comme vaincu, non moins par la maladie en tant que telle, que par son incapacité à avoir su la vaincre. Et c'est bien à ce niveau qu'est présente une souffrance avec toutes ses traductions de crainte, de fuite du patient, de burn-out (Feldman, 2001, p. 458-465), sur lesquelles nous aurons à revenir.

Mais ce rapport à l'impuissance des soignants, s'il a permis le développement des soins palliatifs, risque de faire de ces derniers le «lieu rebut» de tout ce dont la médecine contemporaine ne veut plus, avec toute la souffrance que cela peut engendrer pour les soignants qui, s'étant approprié l'approche de la mort, se trouvent dans cette situation paradoxale de ne pas être compris dans les finalités qu'ils poursuivent tout en y étant convoqués par d'autres. En effet, appréhendés comme les «spécialistes» de la souffrance et de la mort dans un lieu qui n'en veut pas, l'hôpital, environnés d'autres soignants pour qui ces deux réalités sont identifiées comme insoutenables, les soignants en soins palliatifs risquent d'y être enfermés, mandatés par leurs pairs d'un nouveau rapport à la puissance, à l'efficacité, mais à l'égard de ces deux réalités généralement déniées: la souffrance et la mort. Tel est le paradoxe des soins palliatifs, celui de ne pas vouloir être réduits à la seule approche de la souffrance et de la mort 


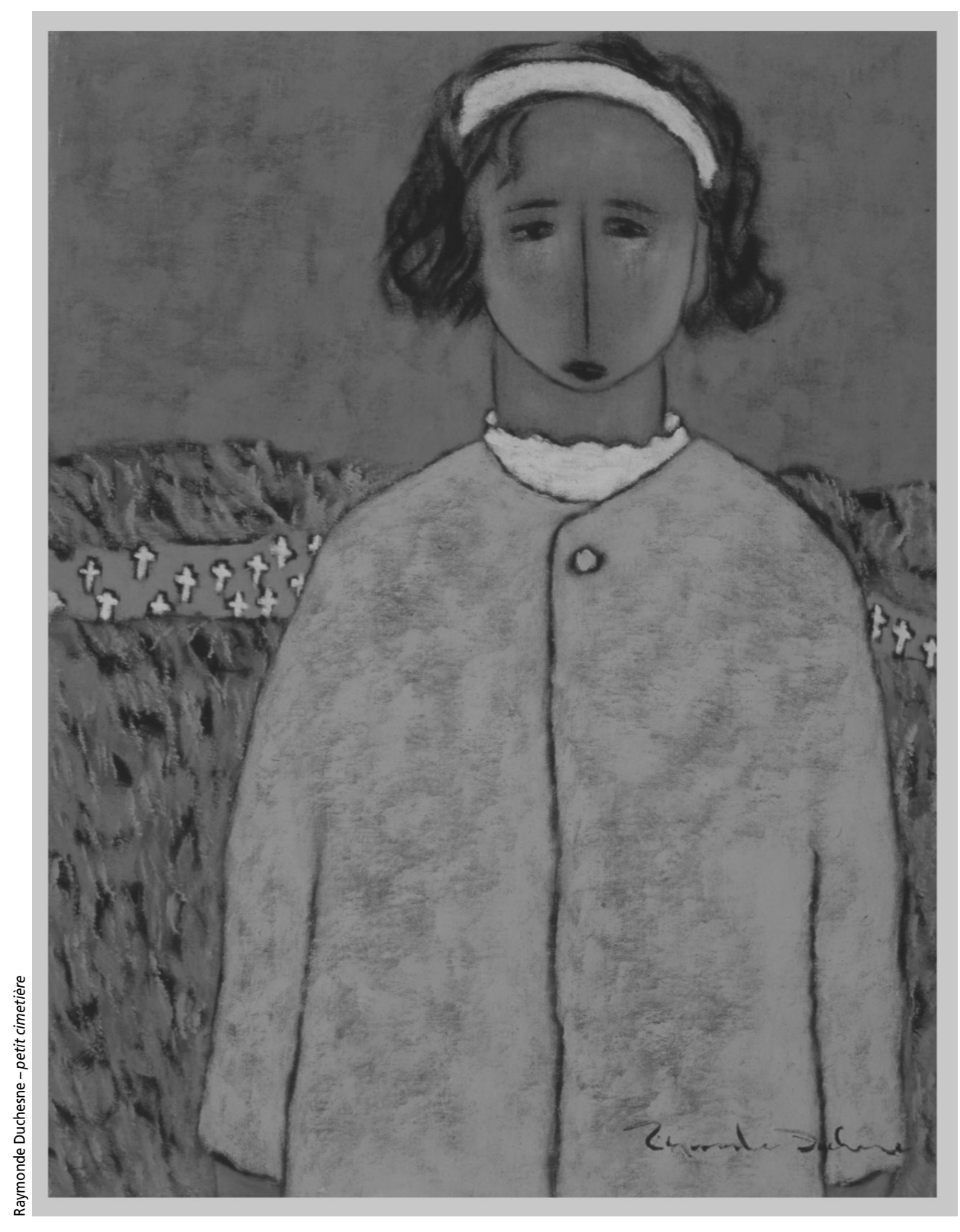

alors que ces dernières sont la première légitimation de développement au cœur de l'hôpital contemporain: «C'est bien l'horizon de la mort indépassable, proche ou lointaine, qui fait la spécificité des soins palliatifs, qui fait peur et que l'on veut gommer, supprimer, ou au moins ne pas nommer». (Marin, 2004, p. 62.)

\section{UNE SOUFFRANCE \\ AUX MULTIPLES FACETTES}

Les quelques éléments qui viennent d'être développés indiquent que le soignant de soins palliatifs sera aisément mis dans une posture difficile au cœur de l'hôpital contemporain, position génératrice de souffrances tant par l'objet de sa responsabilité soignante - la souffrance et la mort - que par les incompréhensions auxquelles il devra faire face dans ce lieu de l'efficacité. desquelles le soignant devra structurer un rapport de proximité mais aussi de nécessaire distance. En un mot, nous touchons ici à toutes ces situations de vie évoquées par tant de soignants et qui, par accumulation, par incapacité de pouvoir en parler conduisent à ce phénomène largement décrit: le burn-out (Feldman, 2001, p. 458-465). Au-delà de ce premier niveau lié à des difficultés d'ordre personnel dans la relation intersubjective, on peut mettre au jour une autre strate de souffrance du soignant liée à une relative dépossession dans sa capacité à soigner: ce sont toutes ces situations où le patient en fin de vie résiste lui aussi: patient douloureuxrésistant, non apaisé, non réconcilié, dont la mort tarde à venir. D'une manière ou l'autre, ce sont toutes ces situations de soins où le patient semble échapper aux «trajectoires acceptables de la fin de vie»(Castra, 2003, p. 269) telles que les soignants se l'étaient idéalement construites.

\section{UNE SOUFFRANCE MORALE}

Cette souffrance personnelle du soignant peut devenir une souffrance réellement morale (Jacquemin, 2004b, p. 11), c'est-àdire une souffrance liée à l'expérience d'une relative incapacité éthique à percevoir ce qui est juste, opportun de faire ou non dans la rencontre singulière de la personne souffrante. Nous touchons ici à l'expérience de la moralité du soignant qui, sans exclure les intrications psychologiques de sa perception, est génératrice de souffrance face à l'interrogation incertaine dans l'action; ce sont toutes ces situations où le soignant, faute de moyens, d'espace de parole ou de formation afin de parvenir à argumenter et rendre compte d'une pratique, ne parvient pas à justifier tout ce qu'il doit faire, tout ce qu'il aimerait faire avec toutes les tensions expérimentées entre un idéal du soin et la prise en charge concrète de cette personne malade, souffrante et proche de sa mort.

Cette souffrance morale des soignants se trouvera renforcée par le mandat qui semble leur être confié et dans l'exercice duquel ils éprouvent un décalage entre ce qu'ils vivent au quotidien et une ambiance sociale qui, en même temps, ne leur est pas étrangère: "les soignants sont pris dans le champ social du $\mathrm{XX}^{\mathrm{e}}$ siècle qui accentue ce déni de la mort et sont formés à renforcer ce déni par la croyance dans les idéaux professionnels qui soutiennent l'illusion de la toute-puissance de la science et, par voie de conséquence, de la médecine. La réalité confronte les soignants qui reçoivent des patients atteints de maladies incurables à la répétition de l'impossibilité de guérir, alors que rien dans la vie sociale ou dans la formation professionnelle ne les a préparés à accepter la mort» (Protin, 1995, p. 13). Et c'est là qu'apparaît une source importante 
de souffrance pour les soignants, situés en tension entre un imaginaire social qui semble conduire à une "inexistence» de la mort, qui semble donner à croire que cette dernière n'existe pas alors qu'en même temps, la société leur fait porter tout le «poids pratique», autrement dit, tout le poids et le prix de sa prise en charge. Il importe de bien mesurer cette tension et d'appréhender en quoi ce décalage entre ce rôle social assigné aux soignants en lien avec un imaginaire de la médecine et la pratique du soin, devient source de souffrance pour le soignant, surtout lorsque le lieu hospitalier où cette dernière s'exerce semble ne pas honorer, reconnaître la lourdeur de cette prise en charge, position dans laquelle se trouvent généralement les soignants en soins palliatifs. En effet, la vie hospitalière semble traversée par un double modèle généralement considéré comme inconciliable: un modèle médico-technique et un modèle holistique. Le premier, par souci d'efficacité et d'une nécessaire objectivation, a tendance à fonctionner par lui-même, indépendamment du vécu du malade tandis que le second reçoit le malade dans l'ensemble de ses aspirations. Or, bon nombre de soignants sont, également par leur formation, sensibles à ce double modèle qu'ils ne peuvent conjointement mettre en œuvre dans leur pratique. Ce manque deviendra vite source de souffrance, sinon de tensions au sein des équipes de soins: les uns taxant le premier modèle d'inhumain, les autres stigmatisant le second comme luxe, perte de temps, etc. Cette critique est souvent adressée aux équipes mobiles de soins palliatifs qui, au sein des unités de soins, "ont le temps de soigner autrement». L'autre piste, qui n'est peut-être qu'une conséquence de la première, sera de repérer une souffrance des soignants face à des impératifs qui leur sont adressés et qui apparaissent inconciliables, situation dans laquelle se trouvent de nombreux soignants voulant mettre en œuvre des soins palliatifs sans en avoir les moyens car leur lieu professionnel n'est pas porteur de leur dimension d'engagement: des impératifs techniques et décisionnels qui ne semblent pas appartenir aux soignants qui, paradoxalement, se trouvent cependant proches du patient dans la situation de soins et mieux à même de percevoir ce qui serait le «meilleur soin » pour ce patient. De plus, cette souffrance se trouve encore renforcée par toutes les incantations pas toujours élucidées dans leur contenu - la prise en charge globale, la dignité du patient, sa responsabilité, son autonomie - mais qui semblent privilégier le modèle holistique, du moins au niveau de l'imaginaire, tout en donnant corrélativement au soignant l'impression qu'il ne pourra jamais respecter le modèle de soin qui lui est "assigné» sans qu'il sache réellement par qui et avec quels moyens. Ainsi, cantonné dans la pratique au niveau des exigences techniques, économiques de rentabilité hospitalière, d'équipe ou d'image de marque, le soignant peut ressentir son travail comme une objectivation d'un modèle institué de soins que, par ailleurs, il refuse ou du moins qu'il questionne. Tous ces éléments évoquant la souffrance multiforme des soignants indiquent à souhait combien il importe de développer des pratiques d'accompagnement psychologique et éthique surtout lorsque la pratique du soin les délocalise à ce point d'eux-mêmes, de leur psychisme, de leur rapport aux valeurs.

\section{UNE EXPÉRIENCE}

\section{DE L'EXCÈS DE RESPONSABILITÉ}

FACE À UN IDÉAL DU SOIN INTENABLE

Mais avant d'envisager plus concrètement la question de l'accompagnement des soignants en vue de les restaurer dans leur subjectivité, nous aimerions nous arrêter sur une dimension centrale de cette expérience de la souffrance morale qu'il s'agira d'épauler, ce que nous nommerions volontiers un excès de responsabilité: «ces excès sont souvent perçus à l'occasion d'impasses, de scandales ou d'émotions particulièrement forts» (Boitte et al., 2002, p. 102).

Mais comment définir cette notion d'excès? Cadoré nous en offre une piste de compréhension lorsque, traitant de la rencontre de l'autre humain, qu'il soit souffrant ou non, il indique que cette rencontre va toujours au-delà de ce qui est à l'œuvre dans le seul présent de cette rencontre, « dès lors que l'homme accepte l'irruption face à lui d'un appel toujours excessif à sa capacité de responsabilité. Pour Levinas, cet appel est celui de l'épiphanie du visage d'autrui lors de toute rencontre. Pour Jonas, c'est l'excès de l'avenir indécis auquel se confronte la responsabilité. Or, précisément, il nous semble que la relation interhumaine au cœur de la médecine est particulièrement illustrative de cette double dimension de l'“excès" »(Cadoré, 1994, p. 98).

Or, c'est bien cet «au-delà » qu'il s'agit de pouvoir percevoir et comprendre, vivre dans sa juste mesure. En effet, si la rencontre de l'autre souffrant renvoie le soignant à une responsabilité qu'il doit exercer, cette dernière se vit parfois avec un poids réel, une sorte d'excès tant il n'est pas possible d'avoir la «maîtrise» de la totalité ni des événements, ni des enjeux qui vont devoir présider à telle ou telle décision singulière. Prenons quelques exemples:

- Une demande de soins exprimée parfois au-delà des possibles raisonnables. («Docteur, ne pourriez-vous pas encore faire quelque chose?
Ne pourriez-vous pas tenter telle intervention chirurgicale?»)

- Des objectifs souhaitables divers et contradictoires (mise en place d'une sonde de gastrostomie qui va permettre d'éviter l'apparition d'escarres mais qui va induire une prolongation de la vie, tout en étant perçue, certes par la famille mais parfois par l'équipe soignante, comme un moyen d'alimentation alors qu'elle est prescrite dans un autre horizon thérapeutique et de sens).

- La difficulté de promouvoir l'unification de la personne dans le contexte d'une médecine de plus en plus technique (approche de la réalité de la mort dans un horizon de chimiothérapies palliatives de plus en plus disponibles dans ses bras successifs).

- Des décisions singulières, ponctuelles, ayant d'importantes répercussions collectives, par exemple l'accueil d'un patient atteint de sclérose latérale amyotrophique.

Face à semblables situations où le sens du bien ne se donne pas immédiatement, où une équipe soignante peut éprouver des visées du bien paradoxales, parfois contradictoires, source de souffrance - toutes situations où la médecine s'expérimente mise en échec au regard de ses propres visées du «bien faire»-, il sera important de mettre en œuvre une réflexion éthique afin d'appréhender le motif, le lieu d'inscription de ce qui est vécu comme une décision excédant la responsabilité soignante, excès perçu comme ce qui conduit le soignant au plus loin des repères habituels, de ce qu'il voudrait pouvoir faire et qu'il ne peut accomplir. La finalité de la réflexion éthique consistera ici à donner toute sa place aux enjeux inhérents à une situation singulière nécessitant une décision, tout en la replaçant dans un contexte théorique plus général; il s'agira donc de mettre en œuvre une capacité critique d'argumentation devant permettre de prendre distance par rapport à ce qui est expérimenté comme excessif au niveau de l'immédiateté de l'action. Cet effort de clarification de ce qui est vécu comme un «excès» qualifiera la démarche éthique comme occasion construite d'un recul critique, argumenté de ce qui est en cause dans une situation singulière. Il s'agira, pour une équipe soignante de mettre en œuvre une créativité éthique en assumant, collectivement, une réflexion sur le sens de l'action: qu'est-ce qui va caractériser une action? Pour qui? Pourquoi ? Comment? En assumant quelles limites? Ce qui demande un réel apprentissage, y compris en soins palliatifs d'autant plus que là, comme ailleurs, les soignants se trouvent confrontés à cette volonté de 
puissance, même si elle semble plus immédiatement qu'ailleurs orientée vers le bien du patient:

Si un sentiment est bien répertorié, c'est celui de l'échec, signifié aux médecins par la mort du patient. Pour nous, praticiens de soins palliatifs, qui pensons avoir accepté l'idée de la mort, ce sentiment peut être d'autant plus source de souffrance que le renoncement à une position de toutepuissance devrait nous en protéger. Nous ne réussissons pas chaque fois dans nos objectifs [...] Enfin, malgré tous nos efforts, nous n'arrivons pas toujours à former nos collègues et nous pouvons nous scandaliser quotidiennement devant des pratiques hospitalières ou médicales dont nous sommes, dans une certaine mesure, solidaires. Heureusement, à l'inverse, le fait d'être confronté à l'échec nous préserve de la tentation - ou du fantasme - de la toute-puissance. Car nous pourrions être tentés pour refuser la défaite, de ne faire que des projets réalistes, ne recherchant que ce que nous serions sûrs d'obtenir et, à l'extrême, en soulageant toute douleur, toute souffrance, avec conscience cynique, voire au prix de la vie des malades.

(Marin, 2004, p. 118-119.).

Ces propos assez longs issus d'une réflexion de soignants nous indiquent admirablement que la pratique des soins palliatifs, quelle que soit son excellence, n'échappe pas aux pratiques de vigilance, certes communes à tout engagement soignant, visant à relire les enjeux de l'action, surtout lorsqu'elle se déroule au cœur d'un idéal du soin exigeant et source d'inutiles culpabilités lorsqu'il n'est pas, nous l'avons $\mathrm{vu}$, resitué dans les limites de chaque situation singulière.

\section{LA RESTAURATION DU SUJET SOIGNANT}

Les quelques propos ici développés montrent aisément, et ceci malgré certains discours construits, qu'il n'est pas toujours aisé d'exercer une juste posture professionnelle en soins palliatifs, que toute pratique peut générer de la souffrance, qu'elle soit due à la typologie des patients rencontrés ou à l'exigence de qualité de soin et d'accompagnement que s'assignent les équipes soignantes. Au terme de ce parcours, comment assumer notre question de départ: quelle possibilité de restaurer les soignants dans leur posture d'humanité? Il nous semble que quelques pistes ont pu être ouvertes.

Tout d'abord, il nous semble urgent de pouvoir reconnaître cette souffrance d'hommes et de femmes engagés dans la pratique des soins palliatifs, y compris dans ces lieux privilégiés que peuvent être les unités de soins palliatifs. En effet, si cette pratique de soins se trouve parfois qualifiée par les autres soignants de «luxueuse», c'est vrai qu'elle possède généralement un quota supérieur de soignants pouvant dès lors avoir une plus large disponibilité mais il est en même temps question de ne pas oublier qui sont les personnes objets de leurs soins, des personnes souffrantes, en fin de vie qu'une pratique habituelle de la médecine est heureuse de leur déléguer.

En ce sens, il nous apparaît tout aussi nécessaire que cette souffrance puisse être dite, sans gêne. Même si des femmes et des hommes ont fait ce choix professionnel des soins palliatifs, ils restent, comme tout autre, limités, fragiles, ceci étant vrai à titre personnel ou de vie d'équipe. Et ceci doit pouvoir être reconnu au cœur d'une pratique professionnelle ayant fait le pari du «toujours mieux». Même en soins palliatifs le «mieux» n'est pas toujours possible, tout simplement parce que l'autre, le malade, échappe, ne se laisse pas enfermer dans nos visées du bien; ceci étant particulièrement vrai lorsqu'il est question de le rencontrer, de le soutenir lorsque sa propre mort vient.

Tout ceci nous conduit à une sorte de plaidoyer à ce que les soignants puissent prendre le risque de s'exposer dans leurs fragilités, limites, souffrances, et que tout cela puisse être dit, partagé; ce que Sölle nomme la redécouverte d'une véritable liturgie de la parole donnant accès - et dès lors possibilité de changement - à la souffrance (Sölle, 1992, p. 91). Ceci implique bien sûr qu'ils en aient les moyens par des groupes de parole, des groupes d'éthique clinique, des parcours de formation permanente, reconnaissant par là que la souffrance tant psychique que morale est une conséquence possible, audible de ce type de soins et qu'elle peut être rencontrée et accompagnée. Bien sûr, ces modalités d'accompagnement des soignants existent déjà en de nombreuses institutions mais devraient être davantage développées, non dans un esprit de concession à une souffrance pouvant handicaper le rapport au travail mais bien dans une dynamique de pleine reconnaissance de la difficulté d'une inscription professionnelle résultant d'un imaginaire social et médical relatif à la mort. Ce constat sollicite certes un engagement des institutions hospitalières et de soins, soutenu par un engagement politique en termes de financement. Mais cet engagement ne serait-il pas normal dans la mesure où ces mêmes institutions font porter à une typologie de soignants le poids et le prix d'une médecine objective et technique offrant peu de place aux plus fragilisés que sont les malades souffrants, en fin de vie.

\section{Bibliographie}

BOITTE, P., B. CADORÉ, D. JACQUEMIN et S. ZORRILLA (2002). Pour une bioéthique clinique, Lille, Septentrion Presses universitaires.

CADORÉ, B. (1997). L'éthique clinique comme philosophie contextuelle, Montréal, Fides.

CADORÉ, B. (1994). L'expérience bioéthique de la responsabilité, Namur, Artel/Fides.

CASTRA, M. (2001). Bien mourir. Sociologie des soins palliatifs, Paris, Presses universitaires de France.

FELDMAN, E. (2001). "Le stress des soignants», dans D. JACQUEMIN (dir.), Manuel des soins palliatifs, Paris, Dunod, p. 458-468.

HENDERSON, V. (1969). Principes fondamentaux des soins infirmiers, Genève, Conseil international des infirmières.

JACQUEMIN, D. (2004a). Éthique des soins palliatifs, Paris, Dunod.

JACQUEMIN, D. (2004b). "La souffrance éthique du soignant», Ethica Clinica, n ${ }^{\circ} 35$, septembre 2004, p. 9-14.

JACQUEMIN, D. (2002). Bioéthique, médecine et souffrance. Jalons pour une théologie de l'échec, Montréal, Médiaspaul.

JACQUEMIN, D. et D. MALLET (2001). "Discours et imaginaire des soins palliatifs: nécessité et modalité d'une distance critique ", Les Cahiers de soins palliatifs, vol. $3, \mathrm{n}^{\circ} 1$, automne, p. 19-32.

LONGNEAUX, J.-M. (2004). L'expérience du mal, Namur, Les éditions namuroises.

MARIN, I. (2004). Allez donc mourir ailleurs! Un médecin, l'hôpital et la mort, Paris, Cerf.

MATRAY, B. (2004). La présence et le respect. Éthique du soin et de l'accompagnement, Paris, Desclée de Brouwer.

MATRAY, B. (1995), "Besoins spirituels et accompagnement», Laennec, $\mathrm{n}^{\circ} 2,44^{\mathrm{e}}$ année, décembre, p. 17-22.

PROTIN, M.-J. (1995). «Souffrance des soignants et demande de formation ", Laennec, $\mathrm{n}^{\mathrm{O}} 1,44^{\mathrm{e}}$ année, octobre 1995, p. 13-16.

SÖLLE, D. (1992). Souffrances, Paris, Cerf.

VASSE, D. (1983). Le poids du réel, la souffrance, Paris, Cerf.

VERGELY, B. (1997). La souffrance, Paris, Éditions Folio.

VIMORT, J. (1987). Ensemble, face à la mort. Accompagnement spirituel, Paris, Centurion. 